\title{
Microclima, produção e composição do mosto da 'Syrah' cultivada sob cobertura de plástico
}

\author{
Microclimate, yield and must composition of Syrah grapevine cultivated under plastic cover \\ Mário José Pedro Júnior ${ }^{\star}$, José Luiz Hernandes, Antonio Odair Santos e Antonio Carlos Loureiro \\ Lino
}

Instituto Agronômico de Campinas, Campinas, SP, Brasil. *Autor para correspondência: mariopedrojunior@gmail.com.

Submissão: 18/05/2018 / Aceite: 01/03/2019

\begin{abstract}
RESUMO
Foi feita a caracterização do microclima do vinhedo, das variáveis de produção das videiras e das propriedades físico-químicas do mosto da uva para vinho 'Syrah' cultivada com cobertura e sem cobertura de plástico impermeável (ráfia). As videiras foram sustentadas em Y e manejadas em regime de safra de inverno na região produtora de uvas de Louveira, SP. Durante o período da maturação das uvas foram feitas determinações, na altura dos cachos, da radiação solar e da temperatura do ar. Na colheita foram determinadas as variáveis fitotécnicas (número de ramos, número de cachos, massa dos cachos e produtividade) e físico-químicas do mosto (teor de sólidos solúveis, acidez total, teor de ácido tartárico, antocianinas, polifenóis totais e taninos). Os valores de temperatura máxima do ar foram superiores nas videiras sob cobertura de plástico em comparação às cultivadas a céu aberto enquanto a radiação solar foi mais elevada na condição sem cobertura. A produtividade e o teor de sólidos solúveis totais foram mais elevados para as videiras sob cobertura de plástico. Não foi observada influência da cobertura de plástico impermeável nos teores de antocianina, polifenóis totais e taninos das uvas.
\end{abstract}

PALAVRAS-CHAVE: Vitis vinifera, uva para vinho, sólidos solúveis, acidez total.

\begin{abstract}
The characterization of vineyard microclimate, plant variables and must physicochemical properties of the 'Syrah' wine grape was done for plants cultivated with and without waterproof plastic cover ("ráfia"). The grapevines were trained in $\mathrm{Y}$-shaped trellis and cultivated during the winter growing season in the grape growing region of Louveira-SP, Brazil. During the grape maturation period, measurements of solar radiation and air temperature were taken at cluster height. In the harvest, the plant variables (number of branches, number of clusters, bunch weight and yield) and must physicochemical characteristics (soluble solids content, total titratable acidity, tartaric acid, anthocyanin, total polyphenols and tannins) were determined. Maximum temperature values were higher under plastic cover when compared to the open sky condition while solar radiation values were higher for the open sky condition. Yield and total soluble solids content values were higher for vines grown under plastic cover. No influence of the use of plastic cover was found for the levels of anthocyanin, total polyphenols and tannin of berries.
\end{abstract}

KEYWORDS: Vitis vinifera, wine grape, soluble solids, titratable acidity.

A cultivar Syrah tem sido utilizada pelos viticultores do leste paulista para atender um crescente mercado consumidor proveniente do turismo rural enológico. Essa variedade utilizada para produção de vinhos finos é adaptada a regiões mais quentes, com possibilidade de obtenção de elevados teores de sólidos solúveis, como observado na região sul de Minas Gerais (FAVERO et al. 2008, MOTA et al. 2010, DIAS et al. 2012) e região nordeste do Estado de São Paulo (REGINA et al. 2011).

Porém, alguns problemas advêm da utilização desta cultivar, pois na região, o período de maturação e colheita coincide com meses de elevado índice de chuva prejudicando o acúmulo de açúcares (MOTA et al. 2010, FAVERO et al. 2008, SANTOS et al. 2011) e afeta de maneira negativa a qualidade das uvas para obtenção de vinhos (REGINA et al. 2011).

Para contornar este problema, tem sido utilizada a poda extemporânea, que desloca o ciclo de produção das videiras para obtenção de colheita em meses (junho-julho) com menor índice pluviométrico e 
maior amplitude térmica. Esta técnica tem sido utilizada com sucesso na região cafeeira do sul do estado de Minas Gerais (FAVERO et al. 2008, MOTA et al. 2010) onde teores de sólidos solúveis mais elevados foram obtidos para a cultivar Syrah. Na região nordeste do Estado de São Paulo foram relatados valores superiores a 24ํㅡㄹ Brix para a 'Syrah' quando colhida durante os meses de junho-julho (REGINA et al. 2011), enquanto na região leste do Estado foi obtido valor próximo a 21ํㅡ Brix (SANTOS et al. 2011) e também valores em torno de $20^{\circ}$ Brix para a 'Syrah' cultivada em safras sequenciais de verão e de inverno (PEDRO JÚNIOR \& HERNANDES 2017).

No entanto o sistema de manejo com utilização da poda extemporânea faz coincidir o desenvolvimento vegetativo das videiras com meses de elevada pluviosidade na região (fevereiro-março), favorecendo a ocorrência de doenças fúngicas, principalmente míldio, e prejudicando a produção. Para diminuir o efeito das doenças fúngicas nas videiras tem sido recomendada a utilização do sistema de produção das videiras sob cultivo protegido com coberturas impermeáveis que diminui sua ocorrência, reduz a utilização de defensivos agrícolas e viabiliza a produção de uvas viníferas (MOTA et al. 2010, ROBERTO et al. 2011, CHAVARRIA \& SANTOS 2013). O cultivo protegido em videiras é facilitado pela utilização do sistema de sustentação das plantas em manjedoura por auxiliar na colocação da cobertura impermeável, permitir melhor exposição das folhas e cachos à radiação solar e melhorar a qualidade do produto. $O$ sistema de sustentação em $Y$ tem sido utilizado para produção de uvas para vinho no estado do Rio Grande do Sul (MOTA et al. 2008, CARDOSO et al. 2008, CHAVARRIA \& SANTOS 2013), de Santa Catarina (FALCÃO et al. 2008) e de São Paulo (PEDRO JÚNIOR \& HERNANDES 2017).

Porém tanto modificações no sistema de sustentação das videiras quanto o uso do cultivo protegido provocam alterações microclimáticas no interior do dossel das plantas principalmente na distribuição da radiação solar e na temperatura do ar no entorno dos cachos (CARDOSO et al. 2008, CHAVARRIA \& SANTOS 2009, COMIRAN et al. 2012), fatores que influenciam no acúmulo de compostos qualitativos das bagas da uva.

Considerando-se as vantagens da poda extemporânea, do uso do sistema de sustentação na forma de $\mathrm{Y}$ e do cultivo protegido com material impermeável, foi desenvolvido experimento para caracterizar a influência na alteração do microclima do vinhedo, nos parâmetros fitotécnicos das videiras e nas propriedades físico-químicas do mosto da cultivar de uva para vinho 'Syrah' manejada sem e com cobertura plástica.

O experimento foi realizado durante o inverno de 2017 em vinhedo com nove anos de idade, da cultivar Syrah com plantas sustentadas em $\mathrm{Y}$ e cultivadas sem cobertura de plástico (a céu aberto) e com cobertura de plástico impermeável (ráfia) em Louveira, SP (latitude: $23^{\circ} 04^{\prime}$ 'S; longitude: $46^{\circ} 55^{\prime} \mathrm{W}$ e altitude de $770 \mathrm{~m}$ ). O clima da região segundo a classificação de Köeppen é Cfb. As videiras foram enxertadas no porta-enxerto Paulsen 1103 e o espaçamento foi de 3,0 m entre fileiras e 1,8 m entre plantas, com densidade aproximada de 1850 plantas por hectare.

As videiras foram manejadas em regime de safra de inverno tendo sido efetuada poda média (com cerca de 6 gemas por ramo) durante o mês de fevereiro e a colheita em julho. Durante a safra de verão, com poda efetuada em agosto, todos os cachos das videiras foram suprimidos antes da fase de florescimento. Foram estimuladas apenas duas gemas por ramo com Dormex ${ }^{\circledR}$ a $4 \%$.

Durante o período de maturação das uvas, iniciando-se na mudança de cor, foram feitas medições de radiação solar global e temperatura do ar acima do dossel das plantas e na altura dos cachos das plantas cultivadas sem cobertura e sob cobertura de plástico impermeável. O sensor de radiação solar (Piranômetro Apogee CS 300-L) foi instalado externamente, acima do dossel do vinhedo e tubos solarímetros (TSL, Delta T Devices) foram utilizados abaixo do dossel na altura dos cachos das videiras. A temperatura do ar foi medida com termopares de cobre-constantan instalados em microabrigos (Campbell Scientific) colocados acima do dossel e na altura dos cachos dos ambientes avaliados. Os sensores foram acoplados a sistema de aquisição automática de dados (Datalogger CR10x, da Campbell Scientific) com leituras a cada minuto e registro dos valores diários.

$\mathrm{Na}$ época da colheita foram coletadas em cada tratamento, de forma aleatória, 120 bagas, sendo da parte superior, média e inferior dos cachos na proporção 1/2/1, respectivamente. Posteriormente, a amostra foi dividida em quatro sub-amostras para cada tratamento para determinação do teor de sólidos solúveis (SS) por meio de refratômetro digital com escala de $0-32^{\circ}$ Brix; pH por meio de pHmetro de bancada; acidez total (AT) obtida por titulação do mosto com solução de $\mathrm{NaOH} 0,1 \mathrm{~N}$ até atingir $\mathrm{pH}=8,2$, sendo o resultado de acidez total expresso em mEq.L ${ }^{-1}$ e teor de ácido tartárico (AcT) expresso em \% (JACKSON \& LOMBARD 1993). Ainda, avaliou-se, em 30 plantas, distribuídas aleatoriamente em cada tratamento, o número de ramos e de cachos por planta. Também, foram colhidos 30 cachos para determinação da massa, tendo sido 
a produção estimada pela massa média dos cachos e número de cachos por planta.

Para estimar a influência da cobertura plástica na área foliar foi medida a porosidade do dossel por meio do uso de imagens digitais. A metodologia consistiu em fotografar, com o uso da câmera digital a copa das plantas, de baixo para cima, de modo a se obter na imagem as áreas correspondentes às folhas e ao espaço entre elas (porosidade). Estas imagens foram tratadas no software ImageJ (RASBAND 1997), para o qual foi desenvolvida uma rotina computacional (macro) que transforma a imagem original (RGB) para o espaço de cor $L^{*} a^{*} b^{*}$ que geram 3 imagens, onde: $L^{*}=$ Luminosidade, $a^{*}=$ coordenada vermelho/verde $(+a$ indica vermelho $e-a$ indica verde) e $b^{*}=$ coordenada amarelo/azul ( $+b$ indica amarelo $e-b$ indica azul). A imagem $L^{*}$ é a que possui as informações com alto contraste entre as folhas e céu (porosidade). Esta imagem foi binarizada sendo que os pixels correspondentes ao céu são pretos e os correspondentes às folhas são brancos. A rotina calcula a porcentagem de pretos (porosidade) na imagem.

Para determinação de níveis de antocianinas, polifenóis totais (unidades de absorção por grama) e taninos (UA), foram colhidas amostras compostas por 50 bagas de uva, em cada tratamento. As bagas foram homogeneizadas e, sobre o macerado resultante, foi feita extração com etanol hidratado, sob agitação; posteriormente, adicionou-se HCL $1 \mathrm{M}$ às amostras e fez-se a leitura em espectrofotômetro para antocianinas $(520 \mathrm{~nm})$ e para polifenóis totais $(280 \mathrm{~nm})$ (ILAND et al. 2004). As determinações de taninos totais foram feitas utilizando-se da mesma amostragem anterior, e sobre o macerado homogeneizado procedeu-se à precipitação dos taninos através de metil-celulose, com posterior leitura em espectrofotômetro (280 nm) (SARNECKIS et al. 2006).

Os valores das variáveis microclimáticas, das características fitotécnicas das videiras e físicoquímicas do mosto obtidos nos diferentes sistemas de produção (sem cobertura e sob cobertura de plástico) das videiras foram submetidos à análise variância tendo sido os valores médios comparados pelo teste $t$ ao nível de $5 \%$ de probabilidade de erro. Os resultados estão apresentados na Tabela 1.

Tabela 1. Médias de características microclimáticas do vinhedo, fitotécnicas das videiras e físico-químicas do mosto da uva 'Syrah' cultivada em regime de safra de inverno sob cobertura de plástico impermeável e a céu aberto.

Table 1. Average values of vineyard microclimate, vine yield components and must physicochemical characteristics of the 'Syrah' grapevine cultivated under open sky and plastic overhead cover conditions during the winter growing season.

\begin{tabular}{|c|c|c|}
\hline Variável & Cobertura plástica & Sem cobertura \\
\hline \multicolumn{3}{|l|}{ Características microclimáticas } \\
\hline Temperatura máxima $\left({ }^{\circ} \mathrm{C}\right)$ & $26,0 \mathrm{a}$ & $25,1 \mathrm{~b}$ \\
\hline Temperatura mínima $\left({ }^{\circ} \mathrm{C}\right)$ & $11,9 \mathrm{~ns}$ & 11,8 \\
\hline $\begin{array}{l}\text { Radiação solar ( } \mathrm{MJ} \mathrm{m}^{-2} \text { dia }^{-1} \text { ) } \\
\text { Transmissão da radiação solar (\%) }\end{array}$ & $\begin{array}{l}1,67 b \\
16,3 b\end{array}$ & $\begin{array}{l}3,17 \mathrm{a} \\
31,0 \mathrm{a}\end{array}$ \\
\hline \multicolumn{3}{|l|}{ Características fitotécnicas das videiras } \\
\hline Número de ramos planta-1 & $38,4 \mathrm{~ns}$ & 37,7 \\
\hline Número de cachos planta-1 & $64,7 \mathrm{~ns}$ & 61,4 \\
\hline Massa do cacho $(\mathrm{g})$ & $147,6 \mathrm{a}$ & $127,8 \mathrm{~b}$ \\
\hline $\begin{array}{l}\text { Produção (kg planta-1 }) \\
\text { Produtividade }\left(\mathrm{t} \mathrm{ha}^{-1}\right)\end{array}$ & $\begin{array}{r}9,54 \mathrm{a} \\
17,66 \mathrm{a}\end{array}$ & $\begin{array}{r}7,84 \mathrm{~b} \\
14,51 \mathrm{~b}\end{array}$ \\
\hline Desfolha (GAP frequency) & $17,96 \mathrm{a}$ & $6,99 \mathrm{~b}$ \\
\hline \multicolumn{3}{|c|}{ Características físico-químicas do mosto } \\
\hline Teor de sólidos solúveis ( $\left.{ }^{\circ} B r i x\right)$ & $22,1 \mathrm{a}$ & $20,1 \mathrm{~b}$ \\
\hline Acidez total $\left(\mathrm{mEq} \mathrm{L}^{-1}\right)$ & 92 ns & 91 \\
\hline Ácido Tartárico (\%) & $0,69 \mathrm{~ns}$ & 0,68 \\
\hline Antocianina $\left(\mathrm{mg} \mathrm{g}^{-1}\right)$ & $1,90 \mathrm{~ns}$ & 1,87 \\
\hline Polifenóis totais $\left(\mathrm{UA} \mathrm{g}^{-1}\right)$ & $1,52 \mathrm{~ns}$ & 1,56 \\
\hline Taninos (UA) & $6,09 \mathrm{~ns}$ & 6,07 \\
\hline
\end{tabular}


Em relação ao microclima das videiras verificou-se que as temperaturas máximas na altura dos cachos das videiras, sob cobertura de plástico, foram superiores às observadas em ambiente a céu aberto em $0,9^{\circ} \mathrm{C}$. O efeito da cobertura de plástico na temperatura máxima de vinhedos também foi verificado na região Flores da Cunha, RS (CARDOSO et al. 2008), de Bento Gonçalves, RS (COMIRAN et al. 2012), na região de Jundiaí, SP (PEDRO JÚNIOR et al. 2013) e em Santa Catarina (VANDERLINDE et al. 2016). Para as temperaturas mínimas não foi observada diferença entre os sistemas de manejo (com e sem cobertura de plástico) pelo teste " $t$ ". De maneira semelhante, diferentes autores relatam não haver influência da cobertura de plástico nas temperaturas mínimas de vinhedos manejados sob cultivo protegido em relação ao céu aberto (CHAVARRIA \& SANTOS 2009, COMIRAN et al. 2012, PEDRO JÚNIOR et al. 2013). A transmissão de radiação solar para a altura dos cachos foi, em média para o período de maturação das uvas, 16,3 e $31,0 \%$, respectivamente, para os tratamentos com cobertura de plástico e a céu aberto. Esses valores foram semelhantes aos observados para a 'Moscato Giallo' sustentada em Y (CARDOSO et al. 2008), para a 'Niagara Rosada' sustentada em Y com e sem cobertura de plástico impermeável (PEDRO JÚNIOR et al. 2013), para a 'Niagara Rosada' sustentada em latada sob cobertura de plástico (COMIRAN et al. 2012) e para as cultivares Cabernet Sauvignon e Merlot em espaldeira (VANDERLINDE et al. 2016).

A análise de variância das variáveis fitotécnicas das videiras indicou que seguem distribuição normal pelo teste de Shapiro-Wilk e por meio da comparação das médias pelo teste "t" verificou-se que não houve diferença estatística, ao nível de $5 \%$ de probabilidade de erro, para número de ramos por planta e número de cachos por planta, indicando a uniformidade de condução do vinhedo nos diferentes sistemas de manejo. O número de ramos médio por planta foi de 38,4 e 37,7 respectivamente, para os tratamentos com e sem cobertura de plástico, enquanto no número de cachos por planta variou entre 64,7 e 61,4 (Tabela 1).

Os valores de massa dos cachos, para os diferentes tratamentos, foram mais elevados nas plantas sob cobertura de plástico $(147,6 \mathrm{~g})$ em comparação ao sistema a céu aberto $(127,8 \mathrm{~g})$ tendo sido estatisticamente diferentes. Esses valores foram semelhantes aos obtidos para a 'Syrah' cultivada em Louveira, SP (PEDRO JÚNIOR \& HERNANDES 2017), inferiores aos relatados para a 'Syrah' em ciclo de inverno em Minas Gerais (DIAS et al. 2012) e superiores aos obtidos também na região sul de Minas Gerais (FAVERO et al. 2008). A cobertura plástica no vinhedo pode favorecer a disponibilidade hídrica das plantas pela menor demanda evaporativa influenciando a quantidade de água nas bagas e no aumento da massa dos cachos (CHAVARRIA et al. 2009)

A produção por planta (Tabela 1) foi superior nas videiras cultivadas sob cobertura plástica $(9,54 \mathrm{~kg}$ planta $\left.^{-1}\right)$ em comparação às plantas cultivadas sem cobertura $\left(7,84 \mathrm{~kg}^{\text {planta }}{ }^{-1}\right)$. Esses valores foram semelhantes aos obtidos para a 'Syrah' em safra de inverno em São Paulo (PEDRO JÚNIOR \& HERNANDES 2017) e superiores aos relatados para a 'Syrah', em ciclo de inverno em Minas Gerais (FAVERO et al. 2008, DIAS et al. 2012) provavelmente em função das videiras utilizadas por esses autores serem sustentadas em espaldeira. Nesse trabalho foi efetuada remoção de todos os cachos na safra de verão, como recomendado para safras de inverno na região sul de Minas Gerais (FAVERO et al. 2008, REGINA et al. 2011). Este fato permite que as videiras acumulem mais reservas durante o período da safra de verão expressando maior produção na safra de inverno

A produtividade estimada para os diferentes tratamentos, levando-se em consideração uma população de 1850 plantas por hectare, foi, em média, 17,66 e 14,51 t.ha-1, respectivamente, para as plantas manejadas sob cobertura de plástico e sem cobertura. A maior produtividade observada no tratamento com cobertura plástica deveu-se a maior massa dos cachos (Tabela 1). Esses valores foram semelhantes aos relatados para a 'Syrah' sob cobertura de plástico (PEDRO JÚNIOR \& HERNANDES 2017) e superiores aos observados em Minas Gerais para a 'Syrah' produzida em safra de inverno e

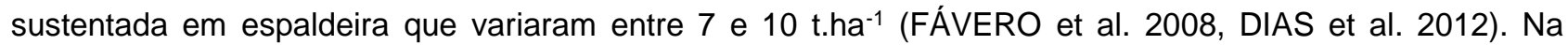
região oeste do Paraná foi observada produtividade superior a 11 t.ha-1 $^{-1}$ para a 'Syrah' sustentada em latada (SATO et al. 2011). O aumento de produtividade em videiras manejadas sob cobertura de plástico também foi observado para a cultivar de uva para vinho Moscato Giallo no Rio Grande do Sul (CHAVARRIA et al. 2009) e para a 'Cabernet Sauvignon' no oeste do Paraná (DETONI et al. 2007) e para videiras 'Niagara Rosada' sob plástico em comparação às cultivadas a céu aberto (COMIRAN et al. 2012). Provavelmente a maior produtividade das videiras sob cobertura de plástico foi relacionada à maior massa dos cachos em comparação às conduzidas a céu aberto.

Os resultados da análise de imageamento (GAP frequency), que podem ser observados na Tabela 1, mostram que as plantas sob cobertura de plástico apresentam uma frequência de vazios menor $(6,99)$, em relação às plantas descobertas $(17,96)$ o que indica um índice de área foliar mais elevado na área protegida pela ráfia. Esta diferença observada na frequência de vazios, provavelmente foi responsável pelas 
diferenças obtidas nos parâmetros de produção e qualidade da 'Syrah'. Também foi verificado que a cobertura com material plástico promoveu incremento da área foliar e da duração do período com área foliar em Niágara Rosada na região de Bento Gonçalves (COMIRAN et al. 2012) e de maneira semelhante, maior massa seca foliar foi obtida em videiras 'Cabernet Sauvignon' cultivadas sob cobertura de plástico (MOTA et al. 2008).

Em relação às características físico-químicas do mosto foi observada diferença estatística nos valores de SS na comparação entre os tratamentos. Para as plantas sob cobertura de plástico os valores de SS foram estatisticamente diferentes e superiores aos observados para a condição a céu aberto. Em média os valores de SS foram: 22,1 e $20,1^{\circ}$ Brix, respectivamente, para a cobertura de plástico e a céu aberto. Apesar de não ter sido observado aumento no de teor de sólidos solúveis para a 'Cabernet Sauvignon' sob cobertura de plástico (DETONI et al. 2007), o uso de cultivo protegido tem sido recomendado por controlar as adversidades climáticas, principalmente o excesso de chuvas (VANDERLINDE et al. 2016). Os valores de SS obtidos nesse trabalho para a 'Syrah' foram semelhantes aos relatados para o sul de Minas Gerais (FAVERO et al. 2008, MOTA et al. 2010, DIAS et al. 2012) e inferiores aos obtidos para a região nordeste do estado de São Paulo cujos teores de açúcar em regime de safra de inverno foram $26,9^{\circ}$ Brix (REGINA et al. 2011). A elevação da temperatura máxima no ambiente sob cobertura plástica eleva a soma térmica, provavelmente encurtando os estádios fenológicos e antecipando o aumento do teor de açúcares nas uvas (VANDERLINDE et al. 2016).

A acidez total e o teor de ácido tartárico não diferiram estatisticamente entre si na comparação das médias entre os tratamentos. Os valores de AT foram da ordem de $90 \mathrm{mEq.L^{-1 }}$ (Tabela 1 ) tendo sido semelhantes aos relatados para a 'Syrah' no sul de Minas Gerais (FAVERO et al. 2008, MOTA et al. 2010) e para a 'Syrah' na região de Louveira, SP (PEDRO JÚNIOR \& HERNANDES 2017). O teor de ácido tartárico nos diferentes tratamentos variou entre 0,68 e $0,69 \%$ tendo sido inferior ao obtido para a 'Syrah' cultivada no Paraná cujo valor médio foi 2,4\% (SATO et al. 2011). Enquanto para a 'Syrah' cultivada no sul de Minas Gerais foi obtido teor de ácido tartárico variando entre 5,29 a $6,36 \mathrm{~g} \cdot \mathrm{L}^{-1}$ (DIAS et al. 2012) e de 5,6 g.L $\mathrm{L}^{-1}$ (MOTA et al. 2010). Os valores de AT e AcT obtidos neste trabalho são considerados adequados à elaboração de vinhos finos.

Os valores médios de antocianinas, polifenóis totais e taninos obtidos para os diferentes tratamentos com exposição à radiação solar diferenciada mostraram semelhança entre as uvas produzidas sob cobertura de plástico e sem cobertura (Tabela 1), mostrando que, a redução da radiação solar global pela presença da cobertura plástica não teve influência no balanço de radiação, a ponto de alterar os níveis de pigmentação fenólica presentes na baga. Diferente dos resultados obtidos neste trabalho, para a 'Cabernet Sauvignon', cultivada em Toledo, PR, foi relatado que o teor de antocianinas totais foi maior nas plantas cultivadas sem cobertura plástica (DETONI et al. 2007), enquanto, para uvas Cabernet Sauvignon e Merlot, sob cobertura plástica em Santa Catarina, os valores de antocianinas e polifenóis foram mais elevados em comparação a área descoberta (VANDERLINDE et al. 2016).

A comparação das características microclimáticas permitiu verificar que a temperatura máxima na altura dos cachos foi mais elevada nas plantas sob cobertura de plástico, enquanto a transmissão de radiação solar foi mais elevada nas plantas cultivadas sem cobertura. Em relação às características fitotécnicas das videiras foi observada maior massa dos cachos e produtividade nas videiras cultivadas sob cobertura de plástico. A respeito das características físico-químicas do mosto da 'Syrah' verificou-se que o teor de sólidos solúveis foi mais elevado nas plantas sob proteção do plástico e a cobertura de plástico não influenciou na acidez total e teor de ácido tartárico e também não alterou os teores de antocianinas, polifenóis totais e taninos das bagas.

\section{AGRADECIMENTOS}

Os autores agradecem à Vinícola Micheletto pelas facilidades proporcionadas em permitir a utilização do vinhedo como área experimental; ao apoio financeiro do Convênio Prefeitura de Louveira - IAC FUNDAG e ao CNPq pela Bolsa de Pesquisa ao primeiro autor (Processo: 302162/2016-0).

\section{REFERÊNCIAS}

CARDOSO LS et al. 2008. Alterações micrometeorológicas em vinhedos pelo uso de coberturas de plástico. Pesquisa Agropecuária Brasileira 43: 441-447.

CHAVARRIA G et al. 2009. Potencial produtivo de videiras cultivadas sob cobertura de plástico. Pesquisa Agropecuária Brasileira 44: 141-147.

CHAVARRIA G \& SANTOS HP. 2013. Cultivo protegido da videira: manejo fitossanitário, qualidade enológica e impacto ambiental. Revista Brasileira de Fruticultura 35: 910-918. 
CHAVARRIA G \& SANTOS HP. 2009. Manejo de videiras sob cultivo protegido. Ciência Rural 39: 1917-1924.

COMIRAN F et al. 2012. Microclima e produção de videiras 'Niágara Rosada' em cultivo orgânico sob cobertura plástica. Revista Brasileira de Fruticultura 34: 152-159.

DETONI AM et al. 2007. Produtividade e qualidade da uva 'Cabernet Sauvignon' produzida sob cobertura de plástico em cultivo orgânico. Revista Brasileira de Fruticultura 29: 530-534.

DIAS FAN et al. 2012. Videira 'Syrah' sobre diferentes porta-enxertos em ciclo de inverno no sul de Minas Gerais. Pesquisa Agropecuária Brasileira 47: 208-215.

FALCÃO LD et al. 2008. Maturity of Cabernet Sauvignon berries from grapevines grown with two different training systems in a new grape growing region in Brazil. Ciencia e Investigatión Agraria 35: 321-332.

FAVERO AC et al. 2008. Viabilidade de produção da videira 'Syrah', em ciclo de outono inverno, na região de Minas Gerais. Revista Brasileira de Fruticultura 30: 685-690.

ILAND P et al. 2004. Chemical analysis of grapes and wine: techniques and concepts. Adelaide: Tony Kitchener Printings. 110p.

JACKSON DI \& LOMBARD PB. 1993. Environmental and management practices affecting grape composition and wine quality - A Review. American Journal of Enology and Viticulture 44: 409-430.

MOTA CS et al. 2008. Comportamento vegetativo e produtivo de videiras 'Cabernet Sauvignon' cultivadas sob cobertura plástica. Revista Brasileira de Fruticultura 30: 148-153.

MOTA RV et al. 2010. Composição físico-química de uvas para vinho fino em ciclos de verão e inverno. Revista Brasileira de Fruticultura 32: 1127-1137.

PEDRO JÚNIOR MJ et al. 2013. Microclima em vinhedos de 'Niagara Rosada' em diferentes sistemas de condução durante safras de inverno e de verão. Revista Brasileira de Fruticultura 35: 151-158.

PEDRO JÚNIOR MJ \& HERNANDES JL. 2017. Produtividade e características físico-químicas da Syrah sustentada em Y durante safra de inverno. Revista Brasileira de Viticultura e Enologia 9: 34-41.

RASBAND WS. 1997. ImageJ. National Institute of Health, Bethesda, Maryland, USA. Disponível em: https://imagej.nih.gov/ij/. Acesso em: 12 out. 2016.

REGINA MA et al. 2011. Caracterização físico-química de uvas viníferas cultivadas em regime de dupla-poda no nordeste do estado de São Paulo. Revista Brasileira de Viticultura e Enologia 3: 84-92.

ROBERTO SR et al. 2011. Revisão: Cultivo protegido em viticultura. Ciência e Técnica Vitivinícola 26: 11-16.

SATO AJ et al. 2011. Fenologia, produção e composição do mosto da 'Cabernet Sauvignon' e 'Tannat' em clima subtropical. Revista Brasileira de Fruticultura 33: 491-499.

SANTOS AO et al. 2011. Parâmetros fitotécnicos e condições microclimáticas para a videira vinífera conduzida sob dupla poda sequencial. Revista Brasileira de Engenharia Agrícola 15: 1251-1256.

SARNECKIS CJ et al. 2006. Quantification of condensed tannins by precipitation with metyl cellulose: development and validation of an optimized tool for grape and wine analysis. Australian Journal of Grape and Wine Research 12: 39-49.

VANDERLINDE G et al. 2016. Composição química das uvas Cabernet Sauvignon e Merlot sob cobertura plástica em Santa Catarina. Revista Brasileira de Viticultura e Enologia 8: 34-42. 